\title{
Slow Elevation in Protein C Activity without a PROC Mutation in a Neonate with Intracranial Hemorrhage
}

\author{
Erika Uehara, $\mathrm{MD}^{1}$ Hiro Nakao, MD ${ }^{1,2}$ Yusuke Tsumura, $\mathrm{MD}^{1}$ Hisaya Nakadate, MD, $\mathrm{PhD}^{2,3}$ \\ Shoichiro Amari, MD ${ }^{4}$ Hideshi Fujinaga, MD, PhD ${ }^{4}$ Yoshiyuki Tsutsumi, MD, PhD \\ Dongchon Kang, MD, $\mathrm{PhD}^{6}$ Shouichi Ohga, $\mathrm{MD}, \mathrm{PhD}^{7}$ Akira Ishiguro, MD, $\mathrm{PhD}^{1,3}$
}

${ }^{1}$ Department of Postgraduate Education and Training, National Center for Child Health and Development, Tokyo, Japan

2 Department of General Pediatrics and Interdisciplinary Medicine, National Center for Child Health and Development, Tokyo, Japan

${ }^{3}$ Division of Hematology, National Center for Child Health and Development, Tokyo, Japan

${ }^{4}$ Division of Neonatology, National Center for Child Health and Development, Tokyo, Japan

${ }^{5}$ Department of Radiology, National Center for Child Health and Development, Tokyo, Japan

${ }^{6}$ Department of Clinical Chemistry, Graduate School of Medical

Sciences, Kyushu University, Fukuoka, Japan

${ }^{7}$ Department of Pediatrics, Graduate School of Medical Sciences, Kyushu University, Fukuoka, Japan

Am J Perinatol Rep 2018;8:e68-e70.
Address for correspondence Erika Uehara, MD, Department of Postgraduate Education and Training, National Center for Child Health and Development, 2-10-1 Okura, Setagaya-ku, Tokyo, Japan (e-mail: uehara-e@ncchd.go.jp).

\begin{abstract}
Keywords

- neonatal stroke

- hemorrhagic infarction

- protein C deficiency

- PROC mutation

Severe protein C (PC) deficiency leads to purpura fulminans and stroke in newborns. However, the clinical impact of plasma PC activity on the development of neonatal cerebral disease remains elusive. We report a case of hemorrhagic stroke associated with neonatal asphyxia and severe PC deficiency. Plasma PC and protein $S$ activity 7 days after birth was $12 \%$ and $43 \%$, respectively. No PROC mutation was found. PC levels did not exceed $20 \%$ until 2 months of age, even in the absence of consumption coagulopathy or vitamin K deficiency. Neither thromboembolic nor hemorrhagic events occurred during the infusion of activated PC concentrate (twice weekly, up to 68 days after birth). The PC activity levels gradually increased to the standard value for age by 9 months of age. The present case showed that neonatal PC deficiency without a PROC mutation caused an intracranial hemorrhage before a slow increase in PC activity.
\end{abstract}

Protein $\mathrm{C}(\mathrm{PC})$ is an anticoagulant factor synthesized in hepatocytes. Activated PC, augmented by protein S (PS), inhibits coagulation factors Va and VIIIa. PC deficiency leads to thromboembolic events; however, in neonates it often causes purpura fulminans and intracranial hemorrhage. ${ }^{1}$

Neonatal PC deficiency can be divided into the inherited and noninherited types. The inherited type is caused by mutations in the protein $C$ gene (PROC). However, the clinical impact of plasma PC activity on the development of neonatal cerebral disease remains elusive. Little is known about the cause and presentation of neonatal noninherited PC deficiency. ${ }^{2}$ The present case showed that neonatal PC deficiency without a PROC mutation was associated with hemorrhagic stroke before a slow increase in PC activity.

\section{Case Report}

A male infant weighing 2,216 g was born by cesarean section at 36 weeks of gestation due to a previous cesarean section and early labor pains. Fetal growth was normal, and the received

November 17, 2017

accepted after revision

February 12, 2018
DOI https://doi.org/

$10.1055 / \mathrm{s}-0038-1639614$ ISSN 2157-6998.
Copyright $\odot 2018$ by Thieme Medical

Publishers, Inc., 333 Seventh Avenue, New York, NY 10001, USA. Tel: +1(212) 584-4662.
License terms

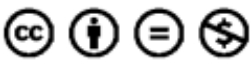



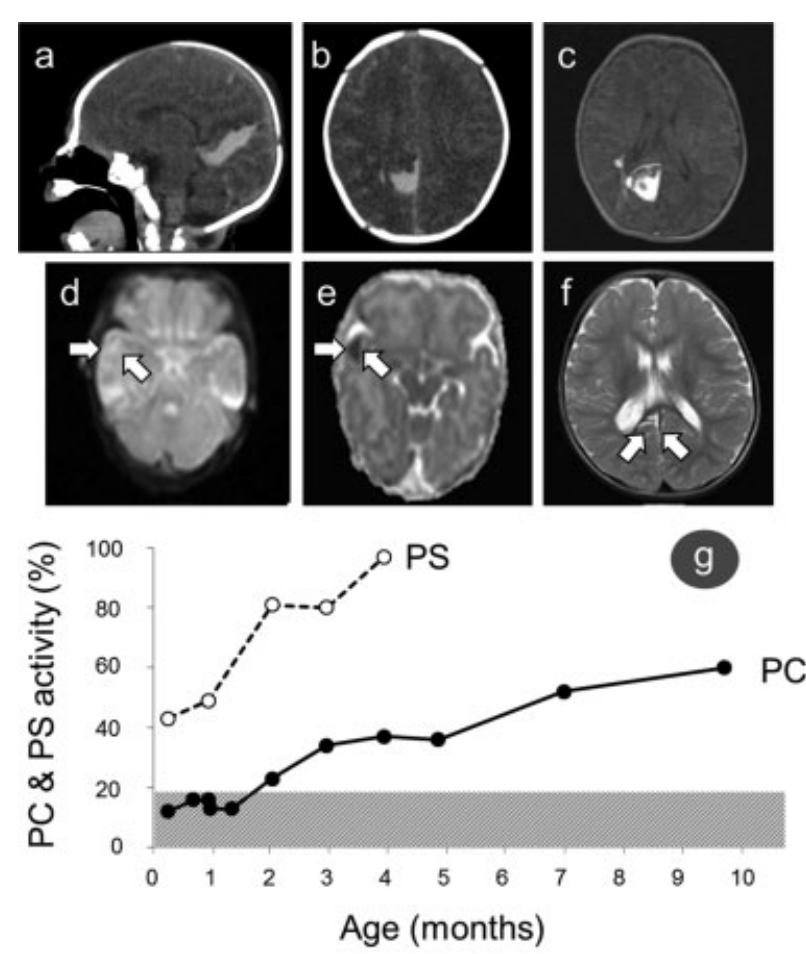

Fig. 1 Intracranial hemorrhage in computed tomograms at 1 day old (A, B) and T1-weighted magnetic resonance imaging at 9 days old (C). A cerebral infarction in diffusion-weighted magnetic resonance imaging (D) and apparent diffusion coefficient map at 9 days old (E). Follow-up T2-weighted magnetic resonance imaging at

18 months old (F) shows no new intracranial embolism or hemorrhage (G) Dissociation between changes in protein $C$ (PC) and protein S (PS) activity. Closed circles and solid lines indicate PC activity; open circles and dotted lines indicate PS activity. PC activity increased slowly compared with the increase in PS activity.

placenta revealed no thromboembolic obstructions. There was no family history of blood coagulation disorder. The patient showed an Apgar score of 2, 4, and 7 at 1, 5, and 10 minutes, respectively.

A subarachnoid hemorrhage, subdural hematoma, and cerebral hemorrhage were found, and ischemic encephalopathy was disclosed by ultrasound and computed tomography at age 1 day. His blood test revealed leukocytes $20.34 \times 10^{9} / \mathrm{L}$, hemoglobin $189 \mathrm{~g} / \mathrm{L}$, platelets $216 \times 10^{9} / \mathrm{L}$, fibrinogen $1.05 \mathrm{~g} / \mathrm{L}$, prothrombin time-international normalized ratio (PT-INR) 1.2, activated partial thromboplastin time (aPTT) 85.6 second, antithrombin activity $37.1 \%$, and Ddimer $27.4 \mu \mathrm{g} / \mathrm{mL}$. The serum creatinine, alanine aminotransferase, and C-reactive protein were normal. A sepsis workup showed sterile culture results for the blood and gastric juice at birth. We infused fresh frozen plasma for 3 days.

At age of 7 days, a coagulation study revealed PC activity at $12 \%(44 \% \pm 80 \%$ at 5 days old and $51 \% \pm 16 \%$ at 1 month old in reference neonates $\left.{ }^{3}\right)$, PS activity at $43 \%(38 \% \pm 14 \%$ at 5 to 7 days old in reference neonates ${ }^{3}$ ), aPTT 67.4 second, and PTINR 1.13 (-Fig. 1). PC and PS activities were assessed by the coagulation method (Instrumentation Laboratory, MA). At 9 days old, magnetic resonance imaging visualized an intra- cranial hemorrhage and multiple peripheral infarctions. Vitamin $\mathrm{K} 2 \mathrm{mg}$ was given intravenously at 1 and 5 days old, and orally at 30 days old. A direct sequencing of the coding and promoter regions of $P R O C$ revealed no mutation at 1 month of age. ${ }^{1}$ There were no further hemorrhagic or thromboembolic events. PC activity slowly increased to adult levels (60\%) by 9 months of age without any thromboembolic events. He learned to roll over at 6 months old, to stand while holding on to objects at 13 months old, and to walk along a wall at 14 months old. A follow-up magnetic resonance imaging at 18 months old showed no new intracranial embolism or hemorrhage.

\section{Discussion}

The present case of neonatal PC deficiency with an intracranial hemorrhage is remarkable because it illustrates the pathophysiology of the disease without a PROC mutation. PC levels in healthy term infants are $30 \%$ to $40 \%$ at age 5 days and $~ 40 \%$ to $50 \%$ at age 1 month. ${ }^{3}$ Levels reach the adult range at about age 6 to 9 months. Manco-Johnson et al showed that the PC activity in some infants increased from a very low level to a normal level, and that some of the infants suffered from thromboembolic events. They also reported that low PC levels occurred most frequently in preterm infants with respiratory distress and infants from twin gestations. ${ }^{4}$ The PC deficiency which occurred in these patients might also occur in stressed neonates like our patient.

There are a few case reports of neonatal noninherited PCdeficient patients born to healthy parents, having normal PC activity, and showing a presentation similar to that of neonates with a PROC mutation. ${ }^{2}$ Two of seven Japanese PC-deficient patients who underwent the PROC gene test had no mutation. ${ }^{1}$ As in our case and a previous case without a PROC mutation, ${ }^{5}$ low PC activity during the early infantile period increased more slowly than the PS activity.

In conclusion, our case of neonatal PC deficiency without a PROC mutation demonstrated an intracranial hemorrhage and slowly increasing PC activity. Further studies are needed to clarify the pathomechanism of neonatal transient PC deficiency.

\section{Statement of Ethics}

The institutional review board of our hospital approved this investigation. Informed consent was obtained from the caretakers for publication of this case report and accompanying images.

\section{Conflict of Interest \\ None.}

\section{Acknowledgments}

This work was supported by grants-in-aid (17929467)(S.O.) from the Japan Agency for Medical Research and Development (AMED), the Research on Measures for Intractable Diseases Project, and the Health and Labor Sciences Research grant (Research on Intractable Diseases) of the Ministry of Health, Labor and Welfare. 
e70 Slow Protein C Activity Rise without a PROC Mutation Uehara et al.

\section{References}

1 Ohga S, Kang D, Kinjo T, et al. Paediatric presentation and outcome of congenital protein C deficiency in Japan. Haemophilia 2013;19 (03):378-384

2 Ohga S, Ishiguro A, Takahashi Y, et al; Japan Childhood Thrombophilia Study Group. Protein C deficiency as the major cause of thrombophilias in childhood. Pediatr Int 2013;55(03): 267-271
3 Takahashi Y, Yoshioka A. Hemostasis and its regulation system in childhood. Jpn J Pediatr Hematol 1994;8:389-397

4 Manco-Johnson MJ, Abshire TC, Jacobson LJ, Marlar RA. Severe neonatal protein $C$ deficiency: prevalence and thrombotic risk. J Pediatr 1991;119(05):793-798

5 Matsunaga Y, Ohga S, Kinjo T, et al. Neonatal asphyxia and renal failure as the presentation of non-inherited protein $C$ deficiency. J Perinatol 2013;33(03):239-241 